\title{
SUBSPACES OF THE NONSTANDARD HULL OF A NORMED SPACE
}

BY

\section{WARD HENSON AND L. C. MOORE, JR.}

ABSTRACT. Normed spaces which are isomorphic to subspaces of the nonstandard hull of a given normed space are characterized. As a consequence it is shown that a normed space is $B$-convex if and only if the nonstandard hull contains no subspace isomorphic to $l_{1}$ and a Banach space is super-reflexive if and only if the nonstandard hull is reflexive. Also, embeddings of second dual spaces into the nonstandard hull are studied. In particular, it is shown that the second dual space of a normed space $E$ is isometric to a complemented subspace of the nonstandard hull of $E$.

Preliminaries. Throughout this paper $(E, \rho)$ will denote a normed space over $K$, where $K$ denotes the real.or complex numbers. By * $\pi /$ we denote an enlargement of some set-theoretical structure $M$ which contains $(E, \rho)$. Recall that ${ }^{*} \pi$ is $k$-saturated over $\pi[12]$ if whenever $X$ is in $\mathbb{N}$ and $\mathcal{C}$ is a collection of intemal subsets of ${ }^{*} X$ such that $\operatorname{card}(\mathcal{C})<\kappa$ and $\mathcal{C}$ has the finite intersection property, then $\mathcal{C}$ has nonempty intersection. Throughout this paper we assume that ${ }^{*} \pi$ is at least an $\boldsymbol{K}_{1}$-saturated enlargement of $\eta$.

The basic nonstandard theory of normed spaces can be found in [12] and [4]. Recall $p \in \epsilon^{*} E$ is called $\rho$-finite if ${ }^{*} \rho(p)$ is a finite element of ${ }^{*} R$; the set of $\rho$-finite elements of ${ }^{*} E$ is denoted by fin $\left.\rho^{*} E\right)$. The $\rho$-monad of 0 is defined by

$$
\mu_{\rho}(0)=\left\{p \mid p \epsilon^{*} E \text { and }{ }^{*} \rho(p) \text { is infinitesimal }\right\} .
$$

The nonstandard hull of $(E, \rho)$ is the vector space $\hat{E}$ over $K$ defined by

$$
\hat{E}=\operatorname{fin}_{\rho}\left({ }^{*} E\right) / \mu_{\rho}(0) \text {. }
$$

The canonical mapping of fin ${ }_{\rho}\left({ }^{*} E\right)$ onto $\hat{E}$ is denoted by $\pi$ (or by $\pi_{E}$ if necessary to avoid ambiguity). A norm $\hat{\rho}$ can be defined on $\hat{E}$ by letting $\hat{\rho}(x)$ be the standard part of ${ }^{*} \rho(p)$, where $\pi(p)=x$. Then $(\hat{E}, \hat{\rho})$ is a Banach space over $K$ (since ${ }^{*} \pi$ is $\boldsymbol{K}_{1}$-saturated) which contains $(E, \rho)$ as a subspace by identifying each $x \in E$ with $\pi\left({ }^{*} x\right)$.

Presented to the Society, January 26, 1973 under the title Nonstandard hulls in the theory of Banach spaces; received by the editors June 4, 1973.

AMS (MOS) subject classifications (1970). Primary 02H25, 46BXX; Secondary 26 A98. 
The dual space $E^{\prime}$ of $(E, \rho)$ is also in $M$ and has a nonstandard hull $\left(\widehat{E^{\prime}}, \widehat{\rho^{\prime}}\right)$. We denote by $\pi^{\prime}$ : fin $\rho^{\prime}\left({ }^{*} E^{\prime}\right) \rightarrow \widehat{E}^{\prime}$ the canonical mapping $\pi_{E^{\prime}}$. As is discussed in $\$ 8$ of [4] there is a natural pairing between $\hat{E}$ and $\widehat{E^{\prime}}$ which yields an identification of $\left(\widehat{E^{\prime}}, \widehat{\rho^{\prime}}\right)$ with a subspace of the dual space of $(\hat{E}, \hat{\rho})$. An important fact relating $\left(\widehat{E}^{\prime}, \widehat{\rho}^{\prime}\right)$ and $(\hat{E}, \hat{\rho})^{\prime}$ is the following (Theorem 8.3 of [4]).

Theorem (retraction theorem). Assume ${ }^{*} \Pi$ is $\kappa$-saturated. If $H$ is a subspace of $\hat{E}$ with Hamel dimension less than $\kappa$ and $\phi \in(\hat{E}, \hat{\rho})^{\prime}$, then there exists $\psi \in\left(\widehat{E^{\prime}}, \widehat{\rho^{\prime}}\right)$ such that $\phi$ and $\psi$ agree on $H$. Moreover, $\psi$ can be chosen so that $\hat{\rho}^{\prime}(\psi)=\hat{\rho}^{\prime}(\phi)$.

Let $E^{c}$ denote the completion of $E$ with respect to $\rho$ and denote the extension of $\rho$ to $E^{c}$ again by $\rho$. Then $\left(E^{c}, \rho\right)$ is contained in $\pi$ and clearly fin $\left.\rho^{*} E\right)=$ fin ${ }_{\rho}\left({ }^{*} E^{c}\right) \cap{ }^{*} E$. Moreover, for each $p \in$ fin ${ }_{\rho}\left({ }^{*} E\right)$ there exists $r \epsilon$ fin $\rho^{*}(E)$ such that ${ }^{*} \rho(p-r)$ is infinitesimal. Thus $\pi_{E}{ }^{c}$ may be identified with $\pi_{E}$ and $(\hat{E}, \hat{\rho})$ may be identified with $\left(\widehat{E^{c}}, \hat{\rho}\right)$.

As further notation, we recall: if $a, b \epsilon^{*} K$, then $a={ }_{1} b$ means $a-b$ is infinitesimal; if $a \epsilon^{*} K$ is finite, then st(a) is the standard part of $a$ in $K$; if $X \epsilon$ $\pi$, then ${ }^{*}[X]$ is the set $\left\{{ }^{*} x \mid x \in X\right\}$ of all standard el ements of ${ }^{*} X$.

1. Hyperfinite dimensional subspaces of the nonstandard hull. In this section we consider briefly certain subspaces of $(\hat{E}, \hat{\rho})$ which are important for technical reasons and which arise in a variety of contexts. (See Definition 1.1.) In this paper we use them in the proof of Theorem 3.4, which concems the structure of the subspaces of $(\hat{E}, \hat{\rho})$.

Let $\mathcal{S}$ be the collection of all finite dimensional subspaces of $E$. In keeping with the usual conventions, we will refer to the elements of ${ }^{*} \delta$ as ${ }^{*}$-finite dimensional subspaces of ${ }^{*} E$. Not only is each element $S$ of ${ }^{*} \delta$ a subspace of ${ }^{*} E$ over ${ }^{*} K$, but if $A$ is any ${ }^{*}$-finite subset of $S$ and $\lambda: A \rightarrow{ }^{*} K$ is any internal function, then the ${ }^{*}$-finite sum

$$
\sum\{\lambda(p) p \mid p \in A\}
$$

is also in $S$. Moreover, there is a ${ }^{*}$-finite subset $B$ of $S$ such that for each $q \in S$ there is a unique intemal function $\lambda: B \rightarrow{ }^{*} K$ such that

$$
q=\sum\{\lambda(p) p \mid p \in B\}
$$

Such a set $B$ will be called a ${ }^{*}$-basis for $S$ over ${ }^{*} K$. In particular if $p_{1}, p_{2}, \cdots$, $p_{n}$ is any finite sequence $(n \in N)$ in ${ }^{*} E$ then the collection of all sums $\sum_{i=1}^{n} \lambda_{i} p_{i}$ where $\lambda_{1}, \lambda_{2}, \cdots, \lambda_{n} \in{ }^{*} K$, is a ${ }^{*}$-finite dimensional subspace of ${ }^{*} E$.

Definition 1.1. A subspace $H$ of $E$ is said to be hyperfinite dimensional 
if there is a * -finite dimensional subspace $S$ of ${ }^{*} E$ such that $H=\pi\left(S \cap\right.$ fin $\left.\rho\left({ }^{*} E\right)\right)$. An important method for obtaining hyperfinite dimensional subspaces of $\hat{E}$ is the following. Let $F_{1}, F_{2}, \cdots$ be a sequence of finite dimensional subspaces of $E$ and $\omega \epsilon^{*} N$. Then ${ }^{*} F_{\omega}$ is a ${ }^{*}$-finite dimensional subspace of ${ }^{*} E$, and hence $\pi\left({ }^{*} F_{\omega} \cap\right.$ fin $\left.\left.\rho^{(}{ }^{*} E\right)\right)$ is a hyperfinite dimensional subspace of $\hat{E}$. In particular, if $(E, \rho)$ is the sequence space $l_{p}$ over $K(1 \leq p \leq \infty)$ and $F_{n}$ is the subspace $l_{p}(n)$ which consists of all sequences $\left(x_{1}, x_{2}, \cdots\right)$ in $l_{p}$ with $x_{n+1}=x_{n+2}=\cdots, 0$, then we will denote the hyperfinite dimensional subspace

$$
\left.\pi^{*} l_{p}(\omega) \cap \operatorname{fin}_{\rho}\left({ }^{*} l_{p}\right)\right)
$$

of $\hat{l}_{p}$ by $\hat{l}_{p}(\omega)$ for $\omega \epsilon^{*} N$. The spaces $\hat{l}_{2}(\omega)$ for $\omega \epsilon^{*} N \sim N$ play an important role in the proof of the invariant subspace theorem due to Robinson and Bernstein. (See [14].) In the present paper we make use of the spaces $\hat{l}_{1}(\omega)$. Note that if $\omega \epsilon^{*} N \sim N$, then $\hat{l}_{1}(\omega)$ contains $l_{1}$.

As was noted in the preliminaries, if $E^{c}$ is the completion of $E$, then the nonstandard hulls $\hat{E}$ and $\widehat{E^{c}}$ may be identified. The next result shows that if this identification is made, then there is no ambiguity in the concept of hyperfinite dimensional subspace.

Lemma 1.2. Let $\left(E^{c}, \rho\right)$ be the completion of $(E, \rho)$ and let $S$ be $a^{*}$-finite dimensional subspace of ${ }^{*} E^{c}$. Then there is $a^{*}$-finite dimensional subspace $S^{\prime}$ of ${ }^{*} E$ such that $\pi\left(S \cap\right.$ fin $\left.\left.\rho^{*}{ }^{*} E^{c}\right)\right)=\pi\left(S^{\prime} \cap\right.$ fin $\left.\left.\rho^{*} E\right)\right)$. Therefore $E$ and $E^{c}$ determine exactly the same hyperfinite dimensional subspaces of $\hat{E}=\widehat{E}^{c}$.

Proof. Recall that for each $n \in N$ there is a positive real number $\alpha(n)$ such that for each $n$-dimensional normed space $(F, \eta)$ with normalized basis $x_{1}, x_{2}$, $\cdots, x_{n}$ (i.e., $\eta\left(x_{1}\right)=\cdots=\eta\left(x_{n}\right)=1$ ) the inequality

$$
\alpha(n) \sum_{i=1}^{n}\left|\lambda_{i}\right| \leq \eta\left(\sum_{i=1}^{n} \lambda_{i} x_{i}\right) \leq \sum_{i=1}^{n}\left|\lambda_{i}\right|
$$

holds for each $\lambda_{1}, \lambda_{2}, \cdots, \lambda_{n} \in K$. Let $\left\{p_{1}, p_{2}, \cdots, p_{\omega}\right\}$ be a ${ }^{*}$-basis for $S$ over ${ }^{*} K$, where $\omega \epsilon^{*} N$. Choose $\delta>0$ in ${ }^{*} R$ so that $\delta /{ }^{*} a(\omega)$ is infinitesimal. Since $E$ is $\rho$-dense in $E^{c}$, there is an intemal sequence $\left\{q_{1}, q_{2}, \cdots, q_{\omega}\right\}$ in ${ }^{*} E$ so that, for each $i=1,2, \cdots, \omega,{ }^{*} \rho\left(p_{i}-q_{i}\right)<\delta$.

Let $\lambda_{1}, \lambda_{2}, \cdots, \lambda_{\omega}$ be any intemal sequence in ${ }^{*} K$. Then

$$
{ }^{*} a(\omega) \sum_{i=1}^{\omega}\left|\lambda_{i}\right| \leq{ }^{*} \rho\left(\sum_{i=1}^{\omega} \lambda_{i} p_{i}\right) \leq \sum_{i=1}^{\omega}\left|\lambda_{i}\right|
$$

and hence 


$$
* \rho\left(\sum_{i=1}^{\omega} \lambda_{i} p_{i}-\sum_{i=1}^{\omega} \lambda_{i} q_{i}\right) \leq\left(\delta /^{*} \alpha(\omega)\right) *\left(\sum_{i=1}^{\omega} \lambda_{i} p_{i}\right) .
$$

Since $\delta /^{*} a(\omega)={ }_{1} 0$ it follows that $q_{1}, q_{2}, \cdots, q_{\omega}$ are ${ }^{*}$-independent over ${ }^{*} K$. That is, $\left\{q_{1}, q_{2}, \cdots, q_{\omega}\right\}$ is a *-basis for the *-finite dimensional subspace $S^{\prime}$ of ${ }^{*} E$ which consists of all sums $\sum_{i=1}^{\omega} \lambda_{i} q_{i}$ where $\lambda_{1}, \lambda_{2}, \cdots, \lambda_{\omega}$ is an intemal sequence in ${ }^{*} K$. It follows that

$$
* \rho\left(\sum_{i=1}^{\omega} \lambda_{i} q_{i}-\sum_{i=1}^{\omega} \lambda_{i} p_{i}\right) \leq\left(\delta /{ }^{*} \alpha(\omega)\right)^{*} \rho\left(\sum_{i=1}^{\omega} \lambda_{i} q_{i}\right)
$$

for $\lambda_{1}, \lambda_{2}, \cdots, \lambda_{\omega}$ an internal sequence in ${ }^{*} K$. Therefore $\sum_{i=1}^{\omega} \lambda_{i} p_{i}$ is $\rho$-finite if and only if $\sum_{i=1}^{\omega} \lambda_{i} q_{i}$ is $\rho$-finite, and in that case

$$
* \rho\left(\sum_{i=1}^{\omega} \lambda_{i} p_{i}-\sum_{i=1}^{\omega} \lambda_{i} q_{i}\right)={ }_{1} 0
$$

Thus $\pi\left(S \cap\right.$ fin $\left.\rho_{\rho}\left({ }^{*} E\right)\right)=\pi\left(S^{\prime} \cap\right.$ fin $\left.\left.\rho^{(*} E\right)\right)$, completing the proof.

We close this section with a discussion of two properties of the hyperfinite dimensional subspaces of $(\hat{E}, \hat{\rho})$ which are of interest in their own right.

Theorem 1.3. Each hyperfinite dimensional subspace of $(\hat{E}, \hat{\rho})$ is closed.

Proof. Let $H=\pi\left(S \cap\right.$ fin $\left.\rho\left({ }^{*} E\right)\right)$, where $S$ is a *finite dimensional subspace of ${ }^{*} E$. Let $\left\{\pi\left(p_{n}\right)\right\}$ be a sequence in $H$ which converges in $(\hat{E}, \hat{\rho})$, say to $\pi(p)$. Using the $\boldsymbol{x}_{1}$-saturation of $\left.{ }^{*}\right)$, extend $\left\{p_{n}\right\}$ to an internal function from ${ }^{*} N$ into ${ }^{*} E$. Since ${ }^{*} \rho\left(p_{n}-p\right)={ }_{1} \hat{\rho}\left(\pi\left(p_{n}\right)-\pi(p)\right)$ for $n \in N$, there exists $\omega \epsilon^{*} N \sim N$ such that $p_{\omega} \in S$ and ${ }^{*} \rho\left(p_{\omega}-p\right)={ }_{1} 0$. That is, $\pi(p)=\pi\left(p_{\omega}\right) \in H$ so that $H$ is closed in $(\hat{E}, \hat{\rho})$.

Theorem 1.4. If ${ }^{*} \Pi$ is $\kappa$-saturated and $A \subseteq \hat{E}$ has cardinality less than $\kappa$, then $A$ is contained in some hyperfinite dimensional subspace of $\hat{E}$.

Proof. Since the cardinality of $A$ is less than $\kappa$ there is a set $B \subseteq$ fin $\left.\rho^{*} E\right)$ such that the cardinality of $B$ is less than $\kappa$ and $A=\pi(B)$. Since ${ }^{*} \pi$ is $\kappa$-saturated there is a ${ }^{*}$-finite subset $B^{\prime}$ of ${ }^{*} E$ such that $B \subseteq B^{\prime}$. (See the proof of Lemma 4.2 in [4].) Let $S$ be the ${ }^{*}$-finite dimensional subspace of ${ }^{*} E$ consisting of all sums of the form $\Sigma\left\{\lambda(p) p \mid p \in B^{\prime}\right\}$ where $\lambda$ is any internal function from $B^{\prime}$ to ${ }^{*} K$. Then $\pi\left(S \cap\right.$ fin $\left.{ }^{*}\left({ }^{*} E\right)\right)$ is a hyperfinite dimensional subspace of $\hat{E}$ which contains $A$.

2. Finite representability and super-reflexivity.

Definition 2.1. Let $(F, \eta)$ be a normed space over the field $K$ and let $\lambda \geq 1$. A linear transformation $T$ of $(F, \eta)$ into $(E, \rho)$ is called a $\lambda$-embedding if $\eta(x)$ 
$\leq \rho(T x) \leq \lambda \cdot \eta(x)$ for all $x \in F$. If such a transformation $T$ exists, $(F, \eta)$ is said to be $\lambda$-embeddable in $(E, \rho)$.

It is easy to see that $(F, \eta)$ is $\lambda$-embeddable in $(E, \rho)$ if and only if there is an invertible bounded linear transformation $Q$ of $F$ onto a subspace $G$ of $E$ such that $Q^{-1}$ is a bounded linear transformation of $G$ onto $E$ and $\|Q\|\left\|Q^{-1}\right\|$ $\leq \lambda$.

Definition 2.2. Let $(F, \eta)$ be a normed space over the field $K$ and let $\lambda \geq 1$. Then $(F, \eta)$ is said to be finitely $\lambda$-representable in $(E, \rho)$ if for each finite dimensional subspace $S$ of $F$ and each $\epsilon>0,(S, \eta)$ is $(\lambda+\epsilon)$-embeddable in $(E, \rho)$.

We now characterize the subspaces of the nonstandard hull $(\hat{E}, \hat{\rho})$ which are isomorphic to normed spaces in $M$. It should be noted that if there is a set of cardinality $\kappa$ in $M$, then for each normed space $(F, \eta)$ of cardinality $\kappa$, there is another normed space in $\pi$ which is isometric to $(F, \eta)$. Therefore the following theorem may be viewed as characterizing the subspaces of $(\hat{E}, \hat{\rho})$ which are of cardinality $\kappa$, for any $\kappa$ which is the cardinality of an element of $\mathfrak{M}$. For example the characterization applies to every separable normed space $(F, \eta)$, since $\pi$ always contains a set of cardinality $2^{\boldsymbol{N}_{0}}$.

Theorem 23. Let $(F, \eta)$ be a normed space over the field $K$ such that $(F, \eta)$ is also contained in $M$ and let $\lambda \geq 1$. The following statements are equivalent:

(i) $(F, \eta)$ is $\lambda$-embeddable in $(\hat{E}, \hat{\rho})$.

(ii) $(F, \eta)$ is finitely $\lambda$-representable in $(\hat{E}, \hat{\rho})$.

(iii) $(F, \eta)$ is finitely $\lambda$-representable in $(E, \rho)$.

Proof. Obviously (i) implies (ii).

(ii) implies (iii): Let $S$ be a finite dimensional subspace of $F$ and let $\epsilon>0$. By (ii) there exists a linear map $U$ from $S$ into $\hat{E}$ such that $\eta(x) \leq \hat{\rho}(U x)$ $\leq(\lambda+(\epsilon / 2)) \eta(x)$ for all $x \in S$. Let $T=[(\lambda+2 \epsilon / 3) /(\lambda+\epsilon / 2)] U$. Then

$$
\eta(x)<\hat{\rho}(T x) \leq(\lambda+2 \epsilon / 3) \eta(x)<(\lambda+\epsilon) \eta(x)
$$

for all $x \in S, x \neq 0$.

Let $\left\{x_{1}, x_{2}, \cdots, x_{n}\right\}$ be a basis for $S$ and pick $p_{1}, p_{2}, \cdots, p_{n}$ in fin $\left.{ }_{\rho}^{*} E\right)$ such that $\pi\left(p_{i}\right)=T x_{i}$ for $i=1,2, \ldots, n$. ( $\pi$ is the canonical quotient map of fin $\left.\alpha^{*} E\right)$ onto $\hat{E}_{\text {.) }}$ We will show that the following inequality holds for every sequence $\lambda_{1}, \lambda_{2}, \cdots, \lambda_{n}$ in ${ }^{*} K$ :

$$
{ }^{*} \eta\left(\sum_{i=1}^{n} \lambda_{i}{ }^{*} x_{i}\right) \leq{ }^{*} \rho\left(\sum_{i=1}^{n} \lambda_{i} p_{i}\right) \leq(\lambda+\epsilon)^{*} \eta\left(\sum_{i=1}^{n} \lambda_{i}{ }^{*} x_{i}\right) \text {. }
$$

If $\lambda_{1}=\lambda_{2}=\cdots=\lambda_{n}=0$, then (\#) is obvious. If $\lambda_{1}, \lambda_{2}, \cdots, \lambda_{n}$ are all finite el ements of ${ }^{*} K$, then 


$$
\begin{aligned}
* \eta\left(\sum_{i=1}^{n} \lambda_{i}{ }^{*} x_{i}\right) & ={ }_{1} \eta\left(\sum_{i=1}^{n} \mathrm{st}\left(\lambda_{i}\right) x_{i}\right)<\hat{\rho}\left(\sum_{i=1}^{n} \mathrm{st}\left(\lambda_{i}\right) T x_{i}\right) \\
& <(\lambda+\epsilon) \eta\left(\sum_{i=1}^{n} \mathrm{st}\left(\lambda_{i}\right) x_{i}\right)={ }_{1}(\lambda+\epsilon)^{*} \eta\left(\sum_{i=1}^{n} \lambda_{i}{ }^{*} x_{i}\right),
\end{aligned}
$$

and so (\#) holds in this case. If $\lambda_{1}, \lambda_{2}, \cdots, \lambda_{n} \in{ }^{*} K$ are arbitrary, but not all $0,(\#)$ is proved by applying the previous argument to the sequence $\lambda_{1} / \alpha, \lambda_{2} / \alpha$, $\cdots, \lambda_{n} / \alpha$, where $a=\max \left\{\left|\lambda_{i}\right|: i=1,2, \cdots, n\right\}$.

Pulling (\#) back to $\pi$, there exist $y_{1}, y_{2}, \cdots, y_{n}$ in $E$ such that for every choice of $\lambda_{1}, \lambda_{2}, \cdots, \lambda_{n}$ in $K$

$$
\eta\left(\sum_{i=1}^{n} \lambda_{i} x_{i}\right) \leq \rho\left(\sum_{i=1}^{n} \lambda_{i} y_{i}\right) \leq(\lambda+\epsilon) \eta\left(\sum_{i=1}^{n} \lambda_{i} x_{i}\right) .
$$

Clearly $V\left(\sum_{i=1}^{n} \lambda_{i} x_{i}\right)=\sum_{i=1}^{n} \lambda_{i} y_{i}$ defines a $(\lambda+\epsilon)$-embedding $V$ of $S$ into $E$. Thus (iii) follows.

(iii) implies (i): Suppose (iii) holds and let $G$ be a ${ }^{*}$-finite dimensional subspace of ${ }^{*} F$ which contains ${ }^{*}[F]$ and let $\delta$ be a positive infinitesimal. Then (iii) implies that there exists a ${ }^{*}$-linear mapping $U$ from $G$ into ${ }^{*} F$ such that

$$
{ }^{*} \eta(p) \leq{ }^{*} \rho(U p) \leq(\lambda+\delta){ }^{*} \eta(p) \text { for all } p \in G .
$$

A function $T$ from $F$ into $E$ may be defined by $T(x)=\pi\left(U\left({ }^{*} x\right)\right)$ for $x \in F$ since $U$ maps ${ }^{*}[F]$ into fin $\left(^{*} E\right)$. It follows immediately from $(\nexists)$ that $T$ is a $\lambda$-embedding of $(F, \eta)$ into $(\hat{E}, \hat{\rho})$, proving (i).

Remarks. 1. The assumption in Theorem 2.3 that the normed space $(F, \eta)$ is in $\pi$ can be replaced by the assumption that the cardinality of $F$ is less than $\kappa$, provided that $* M$ is a $\kappa$-saturated enlargement of $M$. Moreover, in that case the conditions (i), (ii), (iii) of Theorem 2.3 are also equival ent to:

(iv) If $H$ is any hyperfinite dimensional subspace of $\hat{E}$ and $E \subseteq H$, then $(F, \eta)$ is $\lambda$-embeddable in $(H, \hat{\rho})$.

2. A well-known theorem of Dvoretzky [2] states that if $(E, \rho)$ is an infinite dimensional normed space over $R$, then $l_{2}$ is finitely l-representable in $(E, \rho)$. Therefore, in such a case the nonstandard hull $(\hat{E}, \hat{\rho})$ necessarily contains an isometric copy of $l_{2}$.

A number of important properties of Banach spaces $(E, \rho)$ can be stated in terms of the class of Banach spaces which are finitely 1-representable in $(E, \rho)$. Using Theorem 2.3, such properties can often be translated into properties of the space $(\hat{E}, \hat{\rho})$ which are somewhat easier to deal with. One such property is super-reflexivity, as introduced by James ([6], [7], [8] and [9]). In the present terminology, $(E, \rho)$ is super-reflexive if every Banach space which is finitely 1-representable in $(E, \rho)$ is reflexive. 
Theorem 2.4. A Banach space $(E, \rho)$ is super-reflexive if and only if the nonstandard hull $(\hat{E}, \hat{\rho})$ is reflexive.

Proof. From Theorem 2.3 it follows that $(\hat{E}, \hat{\rho})$ is finitely 1-representable in $(E, \rho)$. Therefore, if $(E, \rho)$ is super-reflexive, then $(\hat{E}, \hat{\rho})$ is reflexive.

Conversely, suppose $(E, \rho)$ is not super-reflexive, so that there is a nonreflexive Banach space $(F, \eta)$ which is finitely 1 -representable in $(E, \rho)$. If $(F, \eta)$ is in $\pi$, then Theorem 2.3 implies that $(F, \eta)$ is isometric to a subspace of $(\hat{E}, \hat{\rho})$ and hence that $(\hat{E}, \hat{\rho})$ is not reflexive. Now it follows from Theorem 8.5 of [4] that the reflexivity of $(\hat{E}, \hat{\rho})$ is equivalent to a certain standard condition on $(E, \rho)$. In particular, if some nonstandard hull of $(E, \rho)$ is nonreflexive, then the given nonstandard hull $(\hat{E}, \hat{\rho})$ is al so nonreflexive. The proof is completed by noting that there exists an $\hat{N}_{1}$-saturated enlargement of a set-theoretical structure which contains $(E, \rho)$ and $(F, \eta)$ and therefore there is a nonrefl exive nonstandard hull of $(E, \rho)$.

Note that it follows from Theorem 2.4 that if $(E, \rho)$ is isomorphic to a superreflexive Banach space, then $(E, \rho)$ is also super-reflexive.

Theorem 8.5 of [4] states that if $(E, \rho)$ is a real normed space, then the reflexivity of $(\hat{E}, \hat{\rho})$ is equivalent to the following standard condition:

(\#) For some $r \in R, 0<r<1$, and some positive integer $n$, there do not exist finite sequences $\left\{x_{1}, \cdots, x_{n}\right\}$ in $E$ and $\left\{y_{1}, \cdots, y_{n}\right\}$ in $E^{\prime}$ which satisfy

$$
\begin{gathered}
\rho\left(x_{i}\right)=1, \quad \rho^{\prime}\left(y_{j}\right)=1 \quad \text { if } i, j=1,2, \cdots, n, \\
r<<x_{i}, y_{j}>\text { if } 1 \leq j \leq i \leq n, \text { and } \\
0=\left\langle x_{i}, y_{j}>\text { if } 1 \leq i<j \leq n .\right.
\end{gathered}
$$

(If $(E, \rho)$ is a complex space, then the reflexivity of $(\hat{E}, \hat{\rho})$ is equival ent to the condition obtained from (\#) by replacing $\left\langle x_{i}, y_{j}\right\rangle$ everywhere by $\operatorname{Re}\left\langle x_{i}, y_{j}\right\rangle_{\text {. }}$ ) Condition (\#) is one of the conditions listed as (P4) in [6]. The fact that (\#) is equivalent to the super-reflexivity of $(E, \rho)$, which follows immediately from Theorem 2.4 (above) and Theorem 8.5 of [4], was proved in [6]. Note also that if $(E, \rho)$ satisfies $(\#)$, then $(\hat{E}, \hat{\rho})$ also satisfies $(\#)$. Therefore, $(\hat{E}, \hat{\rho})$ is superreflexive if and only if $(\hat{E}, \hat{\rho})$ is reflexive.

3. Embedding second dual spaces in the nonstandard hull. Luxemburg [13] has shown, using Helly's Theorem, that there is a norm-preserving function $T$ from the second dual $E^{\prime \prime}$ into the nonstandard hull $\hat{E}$ such that $T$ preserves the pairing with $E^{\prime}$. (That is, $\langle x, y\rangle=\langle T x, y\rangle$ for all $x \in E^{\prime \prime}$ and $y \in E^{\prime}$.) By using the principle of local reflexivity [11], [10], which is a strengthening of Helly's Theorem, the existence of such a $T$ which is linear and satisfies $T x=x$ for all $x \in E$, can be proved. The precise statement of the result we use, which is 
proved in [10], is the following.

Theorem 3.1 (principle of local reflexivity). Let $E$ be a Banach space, let $U$ and $V$ be finite dimensional subspaces of $E^{\prime \prime}$ and $E^{\prime}$ respectively and let $\delta>0$. Then there is a one-to-one operator $T: U \rightarrow E$ with $T x=x$ for all $x \in E$ $\cap U, f(T e)=e(f)$ for all $e \in U$ and $f \in V$, and also $\|T\| \cdot\left\|T^{-1}\right\|<1+\delta$.

Theorem 3.2. There is a linear isometry $T$ from $E^{\prime \prime}$ into $\hat{E}$ which satisfies $T x=x$ for all $x \in E$ and $\langle x, y\rangle=\langle T x, y\rangle$ for all $x \in E^{\prime \prime}$ and $y \in E^{\prime}$.

Proof. Let $E^{c}$ be the completion of $E$. Since $\left(E^{C}\right)^{\prime \prime}=E^{\prime \prime}$ and $\widehat{E^{c}}=\hat{E}$ (see the preliminaries) we may assume that $E$ is complete. Let $S$ be a *finite dimensional subspace of * $E^{\prime \prime}$ with $S \supseteq{ }^{*}\left[E^{\prime \prime}\right]$ and let $S^{\prime}$ be a *finite dimensional subspace of ${ }^{*} E^{\prime}$ with $S^{\prime} \underline{2}^{*}\left[E^{\prime}\right]$. Also let $\delta$ be any positive infinitesimal in ${ }^{*} R$. By passing the principle of local reflexivity to ${ }^{*} \pi$ it follows that there is an internal function $P: S \rightarrow{ }^{*} E$ which is linear over ${ }^{*} K$ and satisfies

(i) $(1-\delta) \cdot{ }^{*} \rho^{\prime \prime}(q) \leq{ }^{*} \rho(P q) \leq(1+\delta) \cdot{ }^{*} \rho^{\prime \prime}(q)$ for all $q \in S$,

(ii) $P q=q$ for all $q \in S \cap^{*} E$ and

(iii) $\langle q, r\rangle=\langle P q, r\rangle$ for all $q \in S$ and $r \in S^{\prime}$.

Now define $T$ on $E^{\prime \prime}$ by $T(x)=\pi\left(P\left({ }^{*} x\right)\right)$. $T$ is obviously linear and it is an isometry by (i). If $x \in E$, then ${ }^{*} x \in S \cap{ }^{*} E$ so that $T x=x$. If $x \in E^{\prime \prime}$ and $y \in E^{\prime}$, then $x \in S$ and $y \in S^{\prime}$ from which it follows that $\langle T x, y\rangle=\operatorname{st}\left\langle P\left({ }^{*} x\right),{ }^{*} y\right\rangle=\langle x, y\rangle$ which completes the proof.

There is a natural linear mapping $Q: \hat{E} \rightarrow E^{\prime \prime}$ which is defined by letting $Q(x)$ be the restriction of $x$ (as a linear functional on $\widehat{E^{\prime}}$ ) to $E^{\prime}$, for each $x \in \hat{E}$. That is, for each $x \in \hat{E}$ and $y \in E^{\prime},\langle x, y\rangle=\langle Q x, y\rangle$. Let $T: E^{\prime \prime} \rightarrow \hat{E}$ be a linear isometry as in Theorem 3.2. Then, for each $x \in E^{\prime \prime},\langle Q T x, y\rangle=\langle x, y\rangle$ for all $y \in E^{\prime}$, so that $Q T$ is the identity map on $E^{\prime \prime}$. In particular this shows that $Q$ is onto and $\|Q\|=1$. Also it follows that $T Q$ is a projection (of norm 1) from $\hat{E}$ onto $T\left(E^{\prime \prime}\right)$. From this observation the following corollary is immediate.

Corollary 3.3. $E$ is a complemented subspace of $\hat{E}$ if and only if $E$ is a complemented subspace of $E^{\prime \prime}$.

When $* \pi$ is sufficiently saturated it is possible to strengthen Theorem 3.2 in a useful way by putting certain restrictions on the "location" of the image space $T\left(E^{\prime \prime}\right)$.

Theorem 3.4. Assume that * $\pi$ is $\kappa \cdot$ saturated and $\operatorname{card}\left(E^{\prime \prime}\right)<\kappa$. If $H$ is a byperfinite dimensional subspace of $\hat{E}$ and $E \subseteq H$, then there is a linear isometry $T$ from $E^{\prime \prime}$ into $H$ which satisfies $T x=x$ for all $x \in E$ and $\langle x, y\rangle=\langle T x, y\rangle$ for all $x \in E^{\prime \prime}$ and $y \in E^{\prime}$. 
Proof. Let $E^{c}$ be the completion of $E$. Since $\left(E^{C}\right)^{\prime \prime}=E^{\prime \prime}$ and $\widehat{E^{c}}=\hat{E}$, Lemma 1.2 implies that it suffices to prove the theorem under the assumption that $E$ is complete. Let $\mathcal{F}$ be the collection of all finite dimensional subspaces of $E^{\prime \prime}$. Let $S$ be a *-finite dimensional subspace of ${ }^{*} E$ such that $\pi\left(S \cap\right.$ fin $\left.{ }_{\rho}\left({ }^{*} E\right)\right)=H$. For each $n \geq 1, F \in \mathcal{F}, x \in E^{\prime \prime}$ and $y \in E^{\prime}$ let $A(n, F, x, y)$ be the intemal set which consists of all intemal, *-linear functions $P$ from some *-subspace of ${ }^{*} E$ " into $S$ such that

(i) The domain of $P$ contains ${ }^{*} F \cup\left\{{ }^{*} x\right\}$,

(ii) for all $q$ in the domain of $P$,

$$
(1-1 / n)^{*} \rho^{\prime \prime}(q) \leq{ }^{*} \rho(P q) \leq(1+1 / n)^{*} \rho^{\prime \prime}(q)
$$

(iii) if $x \in E$, then ${ }^{*} \rho\left(P\left({ }^{*} x\right)-{ }^{*} x\right)<1 / n$, and

(iv) $\left|{ }^{*} x,{ }^{*} y\right\rangle-\left\langle P\left({ }^{*} x\right),{ }^{*} y\right\rangle \mid<1 / n$.

Assume that $P$ is in the intersection of all the sets $A(n, F, x, y)$, for $n \geq 1$, $F \in \mathcal{F}, x \in E^{\prime \prime}$ and $y \in E^{\prime}$. Then ${ }^{*}\left[E^{\prime \prime}\right]$ is contained in the domain of $P$; moreover, by (ii), $P\left({ }^{*} x\right) \in$ fin ${ }_{\rho}\left({ }^{*} E\right)$ for each $x \in E^{\prime \prime}$. Thus we may define $T$ on $E^{\prime \prime}$ by $T(x)=\pi\left(P\left({ }^{*} x\right)\right)$. $T$ is obviously linear and it is an isometry by the conditions (ii). By conditions (iii), for each $x \in E,{ }^{*} \rho\left(P\left({ }^{*} x\right)-{ }^{*} x\right)={ }_{1} 0$, so that $T(x)=\pi\left(P\left({ }^{*} x\right)\right)=$ $\left.\pi^{*} x\right)=x$. If $x \in E^{\prime \prime}$ and $y \in E^{\prime}$, then the conditions (iv) imply that $\langle T x, y\rangle=$ st $\left.\left\langle P\left({ }^{*} x\right),{ }^{*} y\right\rangle=s t{ }^{*} x,{ }^{*} y\right\rangle=\langle x, y\rangle$. Finally, $P$ maps ${ }^{*}\left[E^{\prime \prime}\right]$ into $S \cap \operatorname{fin}_{\rho}\left(^{*} E\right)$ so that $T$ maps $E$ " into $H$. Therefore $T$ has the desired properties.

It remains to show that the intersection of the sets $A(n, F, x, y)$ is nonempty. We need only show that the collection

$$
\left\{A(n, F, x, y) \mid n \geq 1, F \in \mathcal{F}, x \in E^{\prime \prime} \text { and } y \in E^{\prime}\right\}
$$

has the finite intersection property, since the collection has fewer than $\kappa$ elements and ${ }^{*} \Pi$ is $k$-saturated. Let $F_{1}, \cdots, F_{m}$ be elements of $\mathcal{F}, x_{1}, \cdots, x_{r}$ $\epsilon E^{\prime \prime}, y_{1}, \cdots, y_{k} \in E^{\prime}$ and $n \geq 1$. Let $F$ be the finite dimensional subspace of $E^{\prime \prime}$ which is spanned by $F_{1} \cup \cdots \cup F_{m} \cup\left\{x_{1}, \cdots, x_{r}, y_{1}, \cdots, y_{k}\right\}$. By the principle of local reflexivity (Theorem 3.1) there is a linear map $Q: F \rightarrow E$ such that

(a) $(1-1 / 2 n) \rho^{\prime \prime}(x) \leq \rho(Q x) \leq(1+1 / 2 n) \rho^{\prime \prime}(x)$ for all $x \in F$,

(b) $Q x=x$ for all $x \in F \cap E$, and

(c) $\left\langle x, y_{j}\right\rangle=\left\langle Q x, y_{j}\right\rangle$ for all $x \in F$ and $1 \leq j \leq k$.

Suppose that $z_{1}, \cdots, z_{s}$ is a basis for $F$ over $K$, and choose $p_{1}, \cdots, p_{s} \in S$ so that $\pi\left(p_{i}\right)=Q\left(z_{i}\right)$ for $1 \leq i \leq s$. We may define an internal function $P$ from ${ }^{*} F$ into ${ }^{*} E$ by

$$
P\left(\sum_{i=1}^{s} \lambda_{i}{ }^{*} z_{i}\right)=\sum_{i=1}^{s} \lambda_{i} p_{i}
$$

for each sequence $\lambda_{1}, \cdots, \lambda_{s}$ in ${ }^{*} K$. Then $P$ is linear over ${ }^{*} K$. Also, if $\lambda_{1}$, $\cdots, \lambda_{s}$ are all finite elements of ${ }^{*} K$, then 


$$
{ }^{*} \rho\left(\sum_{i=1}^{s} \lambda_{i} p_{i}-\sum_{i=1}^{s} \lambda_{i}{ }^{*} Q\left(z_{i}\right)\right)={ }_{1} 0 .
$$

Therefore, from properties (a), (b), and (c) above follow these properties of $P$ :

(a') $(1-1 / n)^{*} \rho^{\prime \prime}(q) \leq{ }^{*} \rho(P q) \leq(1+1 / n)^{*} \rho^{\prime \prime}(q)$ for all $q \epsilon^{*} F$,

(b') ${ }^{*} \rho\left(P\left({ }^{*} x_{i}\right)-{ }^{*} x_{i}\right)<1 / n$ if $1 \leq i \leq r$ and $x_{i} \in E$, and

(c') $\left|<^{*} x_{i},{ }^{*} y_{j}\right\rangle-\left\langle P\left({ }^{*} x_{i}\right),{ }^{*} y_{j}\right\rangle \mid<1 / n$ if $1 \leq i \leq r$ and $1 \leq j \leq k$.

This proves that the collection of sets $A(n, F, x, y)$ has the finite intersection property and completes the proof.

Theorem 3.5. Assume that ${ }^{\prime} \Pi$ is $\kappa-$ saturated. If $(F, \eta)$ is a normed space over $K$ and $\operatorname{card}\left(F^{\prime \prime}\right)<\kappa$, then every $\lambda$-embedding of $(F, \eta)$ into $(\hat{E}, \hat{\rho})$ can be extended to a $\lambda$-embedding of $\left(F^{\prime \prime}, \eta^{\prime \prime}\right)$ into $(\hat{E}, \hat{\rho})$.

Proof. Let $T$ be any $\lambda$-embedding of $F$ into $\hat{E}$. Let $\left\{x_{a} \mid \alpha \in I\right\}$ be a Hamel basis for $F$ over $K$. For each $a \in I$, choose $p_{a} \in$ fin $\left.\rho^{(*} E\right)$ such that $\pi\left(p_{a}\right)=$ $T\left(x_{a}\right)$. Since $\operatorname{card}(I)<\kappa$ and $* \pi$ is $k$-saturated, we may extend the function $\left\{p_{a}\right\}$ to an intemal function from ${ }^{*} I$ into ${ }^{*} E$, which we shall still denote by $\left\{p_{a}\right\}$.

For each $n \epsilon^{*} N$ let $A_{n}$ be the intemal set which consists of all *-finite subsets $J$ of ${ }^{*} I$ such that, for each intemal function $y: J \rightarrow^{*} K$,

$$
\begin{aligned}
(1-1 / n)^{*} \eta\left(\sum_{a \in J} \gamma_{a}{ }^{*} x_{a}\right) & \leq{ }^{*} \rho\left(\sum_{a \in J} \gamma_{a} p_{a}\right) \\
& \leq \lambda(1+1 / n)^{*} \eta\left(\sum_{a \in J} \gamma_{a}{ }^{*} x_{a}\right) .
\end{aligned}
$$

Note that if $J$ is a finite subset of $I$, then ${ }^{*} J \in A_{n}$ for each standard integer $n$. Again using the $\kappa$-saturation of ${ }^{*} \pi$, there must exist $\omega \epsilon^{*} N \sim N$ such that ${ }^{*} J$ $\epsilon A_{\omega}$ for all finite subsets $J$ of $I$. Moreover, again by $k$-saturation, there must exist a ${ }^{*}$-finite subset $J_{0}$ of ${ }^{*} I$ such that $J_{0} \in A_{\omega}$ and ${ }^{*}[I] \subseteq J_{0}$.

Now let $S$ be the ${ }^{*}$-finite dimensional subspace of ${ }^{*} F$ which is spanned over ${ }^{*} K$ by $\left\{{ }^{*} x_{a} \mid \alpha \in J_{0}\right\}$ and let $H=\pi\left(S \cap\right.$ fin $\left.\rho_{\rho}\left({ }^{*} F\right)\right)$. Observe that $F \subseteq H$. By Theorem 3.4 there is a linear isometry $Q: F^{\prime \prime} \rightarrow H$ which satisfies $Q x=x$ for $x \in F$. Define $P: S \rightarrow{ }^{*} E$ by

$$
P\left(\sum_{a \in J_{0}} \gamma_{a}{ }^{*} x_{a}\right)=\sum_{a \in J_{0}} \gamma_{a} p_{a}
$$

for each intemal function $\gamma: J_{0} \rightarrow{ }^{*} K$. Since $\left\{{ }^{*} x_{a} \mid a \in J_{0}\right\}$ is a *-basis for $S$, $P$ is a well-defined, *-linear map. By the selection of $J_{0}$,

$$
(1-1 / \omega)^{*} \eta(q) \leq{ }^{*} \rho(P q) \leq \lambda(1+1 / \omega)^{*} \eta(q)
$$

for all $q \in S$. Therefore we may define $\tilde{P}: H \rightarrow E$ by 


$$
\widetilde{P}(x)=\pi(P(q)) \text { where } x=\pi(q), q \in S .
$$

Thus defined, $\widetilde{P}$ is a $\lambda$-embedding of $H$ into $E$. Moreover, if $a \in I$, then $\widetilde{P}\left(x_{a}\right)$ $=\pi\left(P\left({ }^{*} x_{a}\right)\right)=\pi\left(p_{a}\right)=T\left(x_{a}\right)$. Therefore, for each $x \in F, \widetilde{P}(x)=T(x)$. It follows that the composite mapping $\breve{P} Q$ is a $\lambda$-embedding of $F$ "into $E$ which extends $T$.

Corollary 3.6. Assume that ${ }^{*} \pi$ is $\kappa$-saturated. If $F$ is a Banach space which is isomorphic to a complemented subspace of $\hat{E}$ and $\operatorname{card}\left(F^{\prime \prime}\right)<\kappa$, then $F$ is complemented in $F^{\prime \prime}$.

Proof. Let $T: F \rightarrow \hat{E}$ be an isomorphism of $F$ onto a complemented subspace of $\hat{E}$. It may be assumed that $T$ is a $\lambda$-embedding for some $\lambda \geq 1$. By Theorem 3.5, $T$ may be extended to a $\lambda$-embedding $\widetilde{T}$ of $F^{\prime \prime}$ into $\hat{E}$. Then $T(F)=\widetilde{T}(F)$ is complemented in $\widetilde{T}\left(F^{\prime \prime}\right)$, so that $F$ is complemented in $F^{\prime \prime}$.

Remark. As an example of the use of Corollary 3.6, note that if ${ }^{*} \pi$ is $\left(2 x_{0}\right)^{+}$-saturated, then no complemented subspace of $\hat{E}$ can be isomorphic to $c_{0}$.

4. B-convexity. In this section we consider another property of nomed spaces $(E, \rho), B$-convexity, which is expressible in terms of the class of normed spaces finitely 1-representable in $(E, \rho)$. In Lemma 4.2 we give a nonstandard characterization of $B$-convexity and in Theorem 4.3 we use the methods developed in this paper to prove a few known facts about $B$-convexity. It should be noted that throughout this section the sequence spaces $l_{1}$ and $l_{1}(n)$ are always taken over the same field $K$ as $E$.

Definition 4.1. A normed space $(E, \rho)$ is said to be $B \cdot$-convex if for some positive integer $n$ and some $\delta>0$ there do not exist $x_{1}, \cdots, x_{n}$ in $E$ such that $\rho\left(x_{i}\right)=1$ for $i=1, \cdots, n$ and

$$
\rho\left(\sum_{i=1}^{n} \eta(i) x_{i}\right) \geq n-\delta
$$

for all functions $\eta$ mapping $\{1, \cdots, n\}$ into $\{1,-1\}$.

The concept of $B$-convexity was introduced by Beck [1] who showed that a Banach space $(E, \rho)$ is $B$-convex if and only if a certain strong law of large numbers is valid for $E$-valued random variables. Giesy showed that $(E, \rho)$ is $B$ convex if and only if for some positive integer $n$ and some $\delta>0, l_{1}(n)$ is not $(1+\delta)$-embeddable in $(E, \rho)$. (Theorem 5 and Lemma 6 of [3].) If for some $\delta>0$ the space $l_{1}(n)$ is not $(1+\delta)$-embeddable in $(E, \rho)$, then $(E, \rho)$ is said to be uniformly non-l $l_{1}(n)[5]$. Thus $(E, \rho)$ is $B$-convex if and only if it is uniformly non- $l_{1}(n)$ for some $n$, by Giesy's results. A uniformly non- $l_{1}(2)$ Banach space is also called uniformly nonsquare. 
Lemma 4.2. The following are equivalent.

(i) $(E, \rho)$ is $B$-convex.

(ii) $(\hat{E}, \hat{\rho})$ is $B$-convex.

(iii) $(\hat{E}, \hat{\rho})$ does not contain an isometric copy of $l_{1}$.

(iv) $(\hat{E}, \hat{\rho})$ does not contain an isomorphic copy of $l_{1}$.

Proof. The condition which is satisfied when $(E, \rho)$ is $B$-convex can be passed to $\left.{ }^{*}\right) \pi$ and the resulting property of $\left({ }^{*} E,{ }^{*} \rho\right)$ clearly implies that $(\hat{E}, \hat{\rho})$ is also $B$-convex. Conversely, the $B$-convexity of $(\hat{E}, \hat{\rho})$ implies that $(E, \rho)$ is $B$-convex, since $(E, \rho)$ is a subspace of $(\hat{E}, \hat{\rho})$. This shows that conditions (i) and (ii) are equivalent.

If $(\hat{E}, \hat{\rho})$ is $B$-convex, then it is uniformly non- $l_{1}(n)$ for some $n$ and therefore it cannot contain a subspace isometric to $l_{1}$. Conversely, if $(\hat{E}, \hat{\rho})$ is not $B-$ convex, then it contains an isometric copy of $l_{1}(n)$ for each $n$, by Theorem 23. It follows that for $\omega \epsilon^{*} N \sim N,(\hat{E}, \hat{\rho})$ contains a subspace isometric to $\hat{l}_{1}(\omega)$ and hence it contains a subspace isometric to $l_{1}$. This shows that (ii) and (iii) are equivalent.

Obviously (iv) implies (iii). To complete the proof we prove (ii) implies (iv) by noting that a Banach space isomorphic to $l_{1}$ is not $B$-convex.

An important conjecture [3], [5] is that every $B$-convex $B$ anach space is reflexive. Using Lemma 4.2 this conjecture may be given the following equival ent formulation: a nonstandard hull $(\hat{E}, \hat{\rho})$ is reflexive if and only if $(\hat{E}, \hat{\rho})$ contains no subspace isomorphic to $l_{1}$.

In the following theorem we list a few known results on $B$-convex spaces with some brief comments on how these results can be obtained using the nonstandard characterization of $B$-convexity which is contained in Lemma 4.2.

Theorem 4.3. (i) Every uniformly convex normed space is B-convex [3].

(ii) If a normed space is isomorphic to a B-convex space, then it is B-convex [1], [3].

(iii) A normed space $(E, \rho)$ is B-convex if and only if $\left(E^{\prime \prime}, \rho^{\prime \prime}\right)$ is B-convex [3].

(iv) A normed space $(E, \rho)$ is $B \cdot$-convex if and only if $\left(E^{\prime}, \rho^{\prime}\right)$ is B-convex [3].

Proof. (i) If $(E, \rho)$ is uniformly convex, then $(\hat{E}, \hat{\rho})$ is reflexive (Theorem 8.7 of [4]) and so $(\hat{E}, \hat{\rho})$ cannot contain an isometric copy of $l_{1}$. Thus $(E, \rho)$ is $B$-convex by Lemma 4.2.

(ii) This follows immediately from the equivalence of (i) and (iv) in Lemma 4.2 and the fact that isomorphic normed spaces have isomorphic nonstandard hulls.

(iii) This follows from Lemma 4.2 and the fact that $\left(E^{\prime \prime}, \rho^{\prime \prime}\right)$ can be embedded in $(\hat{E}, \hat{\rho})$ (Theorem 3.2). 
(iv) By (iii) it is sufficient to show that if $(E, \rho)$ is $B$-convex, then $\left(E^{\prime}, \rho^{\prime}\right)$ is $B$-convex. If $\left(E^{\prime}, \rho^{\prime}\right)$ is not $B$-convex then $\left(\widehat{E}^{\prime}, \widehat{\rho}^{\prime}\right)$ contains an isometric copy $H$ of $l_{1}$. The dual space of $H$ is isometric to $l_{\infty}$. Hence for each $n$ and $\delta>0$ there is a $(1+\delta)$-embedding of $l_{1}(n)$ into the dual space of $\left(\widehat{E}^{\prime}, \widehat{\rho}^{\prime}\right)$. Using the retraction theorem (see the preliminaries) it can be shown that for each positive integer $n$ and each $\delta>0$ there is a $(1+\delta)$-embedding of $l_{1}(n)$ into $\left(\widehat{E^{\prime \prime}}, \widehat{\rho^{\prime \prime}}\right)$. Thus $\left(\widehat{E}^{\prime \prime}, \widehat{\rho^{\prime \prime}}\right)$ is not $B$-convex so $\left(E^{\prime \prime}, \rho^{\prime \prime}\right)$ is not $B$-convex by Lemma 4.2. By (iii) it follows that $(E, \rho)$ is not $B$-convex, completing the proof.

Note (Added January 1974). It has been announced by R. C. James [Notices Amer. Math. Soc. 21 (1974), A-188] that there exists a $B$-convex Banach space which is not reflexive.

\section{REFERENCES}

1. A. Beck, $A$ convexity condition in Banach spaces and the strong law of large numbers, Proc. Amer. Math. Soc. 13 (1962), 329-334. MR 24 \#A3681.

2. A. Dvoretzky, Some results on convex bodies and Banach spaces, Proc. Internat. Sympos. on Linear Spaces (Jerusalem, 1960), Jerusalem Academic Press, Jerusalem; Pergamon, Oxford, 1961, pp. 123-160. MR 25 \#2518.

3. Daniel $\mathrm{P}$. Giesy, On a convexity condition in normed linear spaces, Trans. Amer. Math. Soc. 125 (1966), 114-146; Additions and corrections, ibid. 140 (1969), 511-512. MR 34 \#4866; 39 \# 1945.

4. C. Ward Henson and L. C. Moore, Jr., The nonstandard theory of topological vector spaces, Trans. Amer. Math. Soc. 172 (1972), 405-435. MR 46 \#7836.

5. Robert C. James, Uniformly non-square Banach spaces, Ann. of Math. (2) 80 (1964), 542-550. MR 30 \#4139.

6. - Some self-dual properties of normed linear spaces, Sympos. on Infinite Dimensional Topology, Ann. of Math. Studies, no. 69, Princeton Univ. Press, Princeton, N.J., 1972, pp. 159-175.

7. MR $46 \# 7866$.

8. - Super-reflexive Banach spaces, Canad. J. Math. 24 (1972), 896-904.

9. Robert C. James and J. J. Schäffer, Super-reflexivity and the girth of spheres, Israel J. Math. 11 (1972), 398-404. MR 46 \#4175.

10. W. B. Johnson, H. P. Rosenthal and M. Zippin, On bases, finite dimensional decompositions and weaker structures in Banach spaces, Israel J. Math. 9(1971), 488506. MR $43 \# 6702$.

11. J. Lindenstrauss and H. P. Rosenthal, The $\bigodot_{p}$ spaces, Israel J. Math. 7 (1969), 325-349. MR 42 \#5012.

12. W. A. J. Luxemburg, A general theory of monads, Applications of Model Theory to Algebra, Analysis, and Probability (Internat. Sympos., Pasadena, Calif., 1967), Holt, Rinehart and Winston, New York, 1969, pp. 18-86. MR 39 \#6244.

13. - On some concurrent binary relations occurring in analysis, Contributions to Non-Standard Analysis, North-Holland, Amsterdam, 1972, pp. 85-100.

14. A. Robinson, Nonstandard analysis, North-Holland, Amsterdam, 1966. MR $34 \# 5680$.

DEPARTMENT OF MATHEMATICS, DUKE UNIVERSITY, DURHAM, NORTH CAROLINA 27706 